\title{
Repenser les catégories de « majorité » et de " minorité » : l'islamisme comme phénomène minoritaire dans les sociétés occidentales
}

Rethinking the categories of 'majority' and 'minority': Islamism as a minority phenomenon in Western societies

Reconsiderar las categorías de "mayoría" y "minoría": el islamismo como

fenómeno minoritario en las sociedades occidentales

\section{Rachad Antonius}

\section{OpenEdition}

\section{Journals}

Édition électronique

URL : https://journals.openedition.org/remi/7238

DOI : $10.4000 /$ remi. 7238

ISSN : $1777-5418$

Éditeur

Université de Poitiers

Édition imprimée

Date de publication : 1 juin 2015

Pagination : 11-30

ISBN : 979-10-90426-25-2

ISSN : 0765-0752

\section{Référence électronique}

Rachad Antonius, «Repenser les catégories de «majorité » et de " minorité » : l'islamisme comme phénomène minoritaire dans les sociétés occidentales ", Revue européenne des migrations internationales [En ligne], vol. 31 - n² | 2015, mis en ligne le 01 juin 2018, consulté le 14 avril 2022. URL : http://journals.openedition.org/remi/7238; DOI : https://doi.org/10.4000/remi.7238 


\title{
Repenser les catégories de " majorité " et de " minorité " : I'islamisme comme phénomène minoritaire dans les sociétés occidentales
}

\author{
Rachad Antonius ${ }^{1}$
}

\section{Introduction}

Ce texte constitue une ébauche d'analyse d'une situation particulière et délicate : celle de l'expression, dans l'espace public des démocraties libérales occidentales, du discours fondamentaliste islamique, des pratiques culturelles et politiques qui l'accompagnent, et des réactions qu'il suscite. Les paradigmes qui abordent ce phénomène, comme relevant avant tout d'un rapport de pouvoir entre une majorité dominante et une minorité qui prend la parole pour défendre son droit de croire, ne permettent pas de saisir certains enjeux essentiels de la question. L'analyse du discours fondamentaliste est source de controverse, car il se développe au sein de minorités dont une partie est marginalisée socialement et économiquement, ce qui soulève des problèmes tant théoriques que de positionnement éthique et politique.

Du côté de l'enjeu éthique, ne risque-t-on pas d'attiser l'islamophobie ${ }^{2}$ en faisant du phénomène islamiste un objet d'examen ? Faut-il parler de la radicalisation islamiste de façon spécifique ou pas ? Comment le faire ${ }^{3}$ ? Certains chercheurs estiment que le simple fait de faire de l'islamisme radical en situation de migration un objet de recherche sociologique est très problématique, surtout dans un contexte de dérapage sécuritaire et de " guerre à la terreur " (Razack, 2011). Ceci nourrirait le sentiment que la probabilité de radicalisation est plus

\footnotetext{
1 Professeur titulaire, département de sociologie, université du Québec à Montréal, C.P. 8888, Succ. Centre-ville, Montréal, Qc. H3C 3P8 ; antonius.rachad@uqam.ca

2 Nous utilisons ce terme dans un sens très général : hostilité (souvent viscérale) envers tout ce qui relève de l'islam, et promotion de politiques discriminatoires envers I'ensemble des musulmans. Définie ainsi, l'islamophobie constitue une forme de racisme, cependant, nous nous inscrivons en faux contre un usage abusif du terme lorsqu'il est employé pour désigner des critiques rationnelles et légitimes des interprétations radicales du dogme et des comportements qui en découlent, elles-mêmes fortement contestées dans les sociétés musulmanes.

3 Parmi les nombreux propos qui nourrissent la droite xénophobe on retiendra : " si la radicalisation est liée aux musulmans, ses dangers découlent de l'immigration musulmane $"$.
} 
élevée parmi les communautés musulmanes que dans le reste de la société, légitimant ainsi des mesures de surveillance discriminatoires. Selon Shereen Razack, les stéréotypes de "l'homme musulman dangereux " et de "la femme musulmane soumise et victime " ont contaminé les tentatives de lutte contre certaines pratiques comme les mariages forcés ou les crimes d'honneur (Razack, 1998 et 2011). Selon elle, l'opprobre jeté sur la radicalisation d'une partie des communautés musulmanes rejaillit inévitablement sur tous les musulmans. Ce courant de pensée, bien représenté en Amérique du Nord, a adopté une posture apologétique réduisant la radicalisation à une pratique religieuse rigoureuse, et estimant que son étude relève d'une forme d'islamophobie.

Or nous croyons que cette attitude n'est pas soutenable d'un point de vue sociologique, et qu'elle est contreproductive d'un point de vue politique.

Du point de vue théorique, nous croyons que la difficulté principale vient des limites imposées à l'analyse par le recours à l'opposition désormais classique entre minorités et majorité. Comme les musulmans en situation de migration constituent une minorité numérique, leurs pratiques culturelles tombent dans la catégorie des pratiques devant être protégées contre I'hégémonie des groupes majoritaires. Ces derniers se cacheraient derrière un universalisme qui ne serait en réalité qu'un ethnocentrisme déguisé, ou plus spécifiquement de I'islamophobie, pour combattre les pratiques religieuses d'un groupe minoritaire. Telle a été l'orientation générale d'un colloque organisé, en novembre 2010, par I'Institut Simone de Beauvoir, à l'université Concordia à Montréal, en réaction au Projet de loi 94 de l'Assemblée nationale du Québec qui visait à interdire le voile intégral lors de la prestation de services dans les institutions gouvernementales. Ce colloque faisait suite à une déclaration datant d'avril 2010, dans laquelle il est dit que :

"Le projet de loi 94 est chauviniste et présente l'image trompeuse d'un Québec ayant atteint l'égalité entre les sexes tout en sous-entendant que les communautés musulmanes sont intrinsèquement oppressives pour les femmes. En tant que féministes, nous sommes engagées à promouvoir et soutenir l'autonomie des femmes ainsi que leur capacité de comprendre et d'articuler leurs expériences d'oppression dans des termes qui leur sont propres. Et c'est en tant que féministes que nous disons NON aux interventions de l'État qui promettent l'égalité entre les sexes aux dépens de l'autonomie des femmes "

(Institut Simone de Beauvoir, 2010).

Cette approche théorique ne permet pas de concevoir et d'analyser deux aspects de la question que nous identifions comme pertinents : d'abord, les formes de domination et d'exclusion développées dans les sociétés musulmanes d'origine des immigrants, lesquelles produisent des phénomènes comme celui du voile intégral, qui sont transposées en migration et liées à des positions minoritaires ; ensuite, les tentatives d'hégémonie d'un courant répressif, à l'origine minoritaire à l'intérieur des sociétés musulmanes, qui a réussi à transformer ses impératifs dogmatiques en signes identitaires, adoptés et intériorisés par une portion non négligeable des groupes cibles auxquels il s'adresse. Ces deux aspects sont à prendre en compte si l'on veut comprendre la dynamique des rapports entre minorités musulmanes et majorité en migration. 
Ce sont ces propositions que nous souhaitons développer et fonder théoriquement par un retour sur le concept de minorité. Ce faisant, notre réflexion débouchera sur une interrogation plus large concernant les positions de majorité ou de minorité et les rapports entre majorité et minorités.

\section{Préalables}

Nous commencerons par aborder la situation minoritaire du Québec au sein du Canada, situation qui constitue le cadre politique dans lequel d'autres rapports entre majorités et minorités vont pouvoir s'articuler et se discuter, avant de nous situer d'abord sur la définition du fait minoritaire et ensuite sur celle de la question du nombre.

\section{Le contexte de la société québécoise}

La société québécoise héberge une majorité démographique historique - les Canadiens français du Québec - qui se trouve cependant en situation minoritaire à plusieurs niveaux. ${ }^{4}$

Si certaines prérogatives politiques sont du ressort de l'Assemblée nationale du Québec, d'autres, les plus structurantes, relèvent des institutions politiques et des intérêts économiques canadiens. La Charte canadienne des droits et libertés, construite sur une conception très individualiste des droits, est une des structures qui empêchent la " majorité " au Québec de légiférer pour préserver ses droits collectifs (Labelle, 2015 ; Chevrier, 2012). Cette majorité est donc soumise à un système politique plus large dans lequel elle est en position minoritaire. Dans la société québécoise, elle voit ses prérogatives contestées "par le bas ", c'est-à-dire par des minorités démographiques qui, du fait de leur alliance avec des structures fédérales plus larges, contribuent à la minorisation de la " majorité " québécoise. Les conséquences de cette situation affectent directement sa capacité à déterminer les politiques de gestion de la diversité les mieux adaptées à son statut de "petite nation " (Boucher et Thériault, 2003). Ainsi, la politique d'interculturalisme caractéristique du système québécois de gestion de la diversité est-elle souvent battue en brèche au profit du multiculturalisme, du fait des multiples influences, formelles et informelles qui opèrent au niveau fédéral sur les politiques nationales, voire municipales, québécoises. À un niveau infra-provincial, la ville de Montréal - du fait de sa composition démographique - devient l'instance officielle (non liée à un groupe ethnique particulier) où la contestation par le bas de l'ordre " majoritaire " au niveau du Québec peut s'institutionnaliser. Pour exemple, le maire de Montréal, Denis Coderre, libéral et fédéraliste, a joué un rôle très important dans la contestation de la Charte dite " de la laïcité " proposée par le gouvernement du Parti Québécois en 2013-2014 (Assemblée nationale du Québec, 2013)

4 Cette question est discutée par Micheline Labelle dans son article « Multiculturalisme, interculturalisme, antiracisme : le traitement de l'altérité ", publié dans ce volume. Aussi nos remarques seront réduites au strict minimum.

5 Le nom exact de la Charte est : Charte affirmant les valeurs de laïcité et de neutralité religieuse de l'État ainsi que d'égalité entre les femmes et les hommes et encadrant les demandes d'accommodement. 
Cette situation brièvement décrite montre cependant que les rapports entre les groupes ethnoculturels du Québec issus de l'immigration et la " majorité " québécoise dite "de souche " envisagés principalement comme des rapports majoritaires/minoritaires ne sont pas pertinents pour rendre compte des dynamiques réelles à l'œuvre sur le terrain.

\section{Minorité démographique/minorité sociologique}

Nous ne tenterons pas ici de définir le fait minoritaire ou la notion de minorité en général, au regard de l'extrême diversité des situations minoritaires. Bernard Voutat et René Knuesel (1997) soulignent la difficulté de trouver une définition suffisamment large pour englober toutes les situations minoritaires, et simultanément suffisamment précise pour permettre des analyses rigoureuses. Dès lors, et suite à nos remarques précédentes sur la position minoritaire du Québec au sein du Canada, nous traiterons surtout, dans la suite de l'article, des groupes minoritaires issus de l'immigration dans les sociétés occidentales. Bien que cela simplifie quelque peu le problème de la définition, les difficultés conceptuelles ne disparaissent pas pour autant.

Une clarification concerne l'opposition généralement faite entre minorité démographique et minorité sociologique. La plupart des auteurs s'entendent pour dire que c'est l'aspect de minorisation, ou de position dominée, qui prime dans l'analyse des rapports entre majorité et minorités, l'aspect quantitatif, ou démographique, n'étant que secondaire. Il s'ensuit qu'une majorité démographique pourrait être une minorité sociologique si elle est dans un rapport de force où elle est dominée, ou mise en situation de " minorisation ", terme renvoyant au statut de mineur. La plupart des auteurs estiment donc que le terme " minorité " doit être pris dans son sens sociologique et non pas démographique. Cependant ceux qui utilisent ce concept dans son sens sociologique se sentent obligés de l'expliquer pour le ramener finalement à celui de " groupe dominé ". J'en donne pour exemple l'excellente ce qu'en dit Pierre-Jean Simon :

"Le concept sociologique de minorité doit donc être nettement démarqué de tout contenu quantitatif - quelles que soient les incidences, qui sont bien sûr par ailleurs à prendre en compte dans les analyses, de cet aspect du nombre. II ne réfère pas à des ensembles collectifs moins nombreux, mais à des ensembles collectifs dominés.

De même que le concept de majorité, dans cette sélective ne signifie pas le plus nombreux, mais le dominant" (Simon, $2006:$ 155)

Si tel est le cas, pourquoi ne pas désigner les groupes en question par les termes de "dominant " et " dominé " ? II serait étrange par exemple, à propos de la situation en Afrique du Sud au temps de I'apartheid, de parler des privilèges de la " majorité blanche " et de la marginalisation de la " minorité noire ", en s'appuyant sur le sens sociologique de ces concepts. II faudrait immédiatement dire que c'est une minorité démographique qui est en position dominante face à une majorité démographique dominée. Et c'est justement là, la spécificité de cette situation qui la rendait moralement inacceptable, du fait des mesures de ségrégation et de répression violente nécessaires au maintien de la domination. C'est-à-dire que pour employer les termes " minorité " ou " majorité " dans leur sens sociologique (qui est contre-intuitif), il faut toujours fournir l'explication que 
l'usage du terme est censé rendre inutile. Même si le terme " minorité " est une métaphore intéressante attirant l'attention sur le rapport de pouvoir et non sur le nombre, les termes de dominant et de dominé communiquent le sens voulu de façon plus claire et plus directe ${ }^{6}$. Nous ferons usage du terme " minorité " pour désigner "des ensembles collectifs moins nombreux ", en signalant les cas où une minorité démographique est en situation de domination. Le vocabulaire français nous permettra aussi de désigner les diverses formes de rapports de pouvoir entre les groupes sociaux : domination, marginalisation, exclusion, incorporation segmentée, contrôle, discrimination, racisation, etc. Ce choix est d'autant plus justifié qu'en général, les groupes issus de l'immigration sont démographiquement minoritaires.

\section{Retour sur les rapports entre majorités et minorités}

La notion de minorité en tant que telle sans égard aux critères de différenciation spécifiques qui la constitue a été relativement peu étudiée en sociologie. II existe pourtant de très nombreuses études sur des minorités spécifiques, en particulier ethniques ou religieuses, au côté desquels quelques travaux fondateurs ébauchent également l'idée d'une sociologie du fait minoritaire. Les débuts d'une telle sociologie peuvent être retracés chez Louis Wirth (Wirth, 1945). Le sociologue américain a bâti une réflexion sur le fait minoritaire plus large que le contexte social spécifique qu'il étudiait. Beaucoup plus tard, les travaux de Colette Guillaumin (1972 ; 1992) ont apporté une contribution importante, démontrant entre autres que l'idéologie raciste a suivi, et non pas précédé, l'institutionnalisation de rapports de pouvoir incarnés dans la grande traite des esclaves vers les Amériques. Ils ont permis la maturation de l'idée d'une liaison intime entre processus de différenciation et de hiérarchisation. PierreJean Simon (2006) intègrera dans son ouvrage tous ces apports et contribuera à fonder une sociologie du fait minoritaire, en s'intéressant notamment aux processus plus généraux de différentiation et de hiérarchisation des groupes sociaux.

L'approfondissement de ces deux processus et de leur rôle structurant dans les rapports entre majorité et minorités est fondamental puisque les politiques d'intégration sont généralement pensées, justifiées et analysées en faisant référence, explicitement ou implicitement, aux catégories de minorité et de majorité et aux rapports de pouvoir qu'elles supposent. Le rapport entre les deux catégories est considéré comme fondateur du cadre régissant la gestion de la diversité. Nous constatons cependant que sa conceptualisation est problématique à plusieurs égards, divers facteurs contribuant à complexifier le rapport de pouvoir entre les groupes désignés par ces deux catégories. II faut donc déconstruire certaines conceptions considérées comme évidentes autour de ces rapports de pouvoir. Nous le ferons en nous centrant sur un type spécifique de minorités, celles issues de l'immigration dans les pays occidentaux, en considérant la situation spécifique du Québec.

6 Les termes "processus de minorisation " ou " situation de minorisation " peuvent être appropriés, car ils permettent de focaliser I'attention sur les rapports de domination. 
Dans le débat public, certaines analyses et prises de position d'intellectuels et d'institutions, au Canada et au Québec, ont été construites sur le fait que des demandes sociales - $d^{\prime}$ accommodement ou de modification des règles de gestion de l'espace public - sont légitimées par le seul fait que, venant d'un groupe minoritaire, elles doivent être accommodées pour préserver les droits fondamentaux de ce groupe. Toute revendication est perçue comme une façon de contester un rapport de pouvoir inéquitable, de " regarder les Blancs dans le blanc des yeux ${ }^{7}$ et doit donc être appuyée s'il y a volonté de corriger l'injustice. La revendication d'instaurer en Ontario des tribunaux d'arbitrage des conflits familiaux sur la base de la charia - dont les décisions seraient officiellement et automatiquement avalisées par le ministère provincial de la Justice - a été appuyée par certains universitaires ${ }^{8}$. L'opposition à cette revendication ou à des revendications similaires, exprimées par des groupes musulmans au nom de la liberté religieuse, a été qualifiée d'islamophobe. II peut s'avérer que ce soit le cas mais d'autres motivations peuvent être légitimement exposées (voir infra).

\section{Les limites de l'approche constructiviste}

Une autre difficulté vient d'un usage non critique de l'approche constructiviste, pour laquelle les catégories ethniques n'existent pas en soi, mais s'édifient surtout à partir de rapports de pouvoir. Cette approche a permis de déplacer I'accent mis sur les marqueurs ethniques et sur les formes d'ethnicité symboliques, considérés précédemment comme les causes et les évidences de la différence, pour souligner que ce sont plutôt les différences de pouvoir entre groupes qui font émerger la signification des marqueurs. Cette idée est explicitée par Pierre-Jean Simon :

"Les hiérarchies sociales [...] ne se fondent pas sur des différences préexistantes.

Elles les utilisent et les manipulent. Et, plus encore, elles les créent, les fabriquent, ces différences socialement produites servant, par effet de feed-back, de rétroaction, à légitimer les hiérarchies. Ainsi la hiérarchie crée au moins autant et, en fait, bien davantage de la différence que la différence ne crée de la hiérarchie. "

(Simon, 2006 : 133).

Cette conceptualisation a le mérite de porter l'attention sur les processus de différenciation qui sont étroitement liés aux processus de hiérarchisation. Pierre-Jean Simon écrit :

"Ce qu'il convient par ailleurs de considérer, c'est que les deux termes de différenciation et de hiérarchisation sont à prendre ensemble, comme un couple conceptuel indissociable. II n'y a pas d'une part la différence, d'autre part la hiérarchie ".

(Simon, 2006 : 133).

Des particularités de l'ordre de l'apparence ou du phénotype sont instrumentalisées par le groupe dominant. Il leur accorde une valeur morale ou intellectuelle, justifiant une hiérarchie au bénéfice des dominants. Bien que le trait caractéristique puisse être présent avant qu'il ne soit articulé à un processus de

7Titre d'un livre de Shereen Razack : Looking White People in the Eye: Gender, Race, and Culture in Courtrooms and Classrooms, Toronto, University of Toronto Press, 1998.

8 Voir Korteweg (2006) pour un résumé succinct des débats et des arguments sur cette question. 
hiérarchisation, c'est ce processus qui lui donne sa visibilité d'abord en le transformant en différence puis sa signification et sa fonction dans la légitimation de la hiérarchie. Ceci est illustré par les représentations dominantes de ce que devrait être une "femme " ou un "Noir américain ", par exemple. Dans cette perspective, la visibilité elle-même devient problématique, et l'officialisation de cette visibilité est le premier pas vers la hiérarchisation.

La différentiation, la hiérarchisation, et le lien entre elles, sont fondamentaux dans la sociologie du fait minoritaire. Or appliquée automatiquement à toutes les situations de minorisation, cette approche a des limites qu'il faut préciser, sans quoi elle peut induire en erreur. II convient donc de mener une réflexion critique le couple différenciation, hiérarchisation qui est au fondement de la conception constructiviste du fait minoritaire. II s'agit de cerner les limites de sa validité, et essayer d'identifier les éléments qui la modulent, ainsi que le rôle de certains marqueurs de la différence. En effet, quand ces marqueurs sont érigés en frontière symbolique visant une différenciation entre les groupes, ce n'est pas toujours par le groupe dominant, et ce n'est pas toujours en réaction au pouvoir du groupe dominant. Dans certains cas, la frontière est érigée par le groupe minoritaire.

Par exemple, comment penser les situations où des groupes minoritaires revendiquent la différence alors que la société " majoritaire " tente de l'ignorer ? ${ }^{9}$ La réponse la plus commune est qu'il s'agit là d'une stratégie de retournement du stigmate considérée comme libératrice, car les dominés retournent contre les dominants les armes symboliques par lesquelles ces derniers marginalisent les premiers. Ceci est non seulement vrai pour les marqueurs de différentiation particuliers, mais aussi pour la notion de " race " elle-même, dont l'usage dans les analyses et dans les luttes antiracistes a fait l'objet de nombreux débats académiques (Labelle, 2010). Or, nous prétendons que ces situations de revendication de la différence par des groupes minoritaires ne relèvent pas toutes d'une stratégie de retournement du stigmate. Ceci est particulièrement vrai quand les marqueurs ne sont pas des phénotypes, mais des comportements incorporés dans la culture des groupes minoritaires alors que des transformations ont lieu en dehors des sociétés d'accueil. Il arrive dans ce type de situation que la hiérarchisation symbolique soit renversée, les minoritaires se réclamant d'une supériorité morale sur la majorité, accompagnée parfois d'un sentiment de mépris qui se manifeste par le désir de maintenir des barrières symboliques les séparant de la majorité. Ce sentiment de supériorité pouvant relever, dans certains cas, de la transposition d'une idéologie de domination, du lieu où le groupe est majoritaire vers un lieu où le groupe est en situation minoritaire.

Concernant les comportements et les discours islamistes, leur transformation en marqueurs de la différence ne relève pas de la société majoritaire. Leur signification de marqueurs de la différence s'est construite dans un processus de différenciation/hiérarchisation dont les effets sont ensuite transposés en situation minoritaire. Une élaboration et des illustrations de ces processus seront discutées dans la dernière partie de ce texte.

9 Voir Appiah et Gutmann (1998) sur le débat américain concernant la notion de " color-blind". 
Notre analyse de ces processus est structurée autour des points principaux suivants : I'échelle à laquelle on se situe, le rôle du groupe jugé minoritaire dans l'édification de ces clivages, la conception même de la notion de majorité, et enfin la logique majoritaire qui peut être trouvé dans le discours de groupes considérés minoritaires. Parmi ces points, le troisième est de nature exploratoire. Nous souhaitons proposer une autre perspective que celle où la majorité est pensée comme groupe social bien circonscrit, et insister sur le rôle du système normatif de référence dans la définition même de la majorité. Cette définition nous permettra de voir autrement la notion de minorité.

Nous allons maintenant examiner ces différents points en prenant comme ancrage la question des musulmans et de l'islam comme phénomène minoritaire dans les pays occidentaux. Ce choix est justifié par la spécificité de cette question et par sa prépondérance dans le débat public sur " l'injonction à s'intégrer $~^{10} \mathrm{~d}^{\prime}$ une part et le " refus d'intégration " de l'autre. Toutefois, cette question soulève des enjeux extrêmement différents en Europe et au Québec.

\section{Question d'échelle}

On peut facilement concevoir une situation où un groupe démographiquement minoritaire localement peut être partie prenante, à une autre échelle, de rapports de pouvoir et faire partie d'un groupe majoritaire. Dans le cas du Québec, deux autres niveaux sont pertinents, selon le clivage qui départage la majorité des minorités. Nous avons parlé plus haut de la situation de la majorité historique francophone, qui est majoritaire à l'échelle du Québec, mais minoritaire à l'échelle du Canada. Nous souhaitons élaborer à présent la situation minoritaire à l'intérieur du Québec.

Si on se situe uniquement à l'échelle du Québec, on peut en conclure que I'anglais est une langue minoritaire et qu'elle doit être protégée, comme le réclament certains groupes anglophones par des démarches tant politiques que juridiques. Si on se place maintenant à l'échelle du Canada ou de l'Amérique du Nord, le clivage linguistique qui fait des anglophones une minorité dans l'espace québécois les situe du côté majoritaire pour certains aspects des rapports de pouvoir. Non seulement à cause du lien avec le Canada anglais qui permet d'invalider certaines lois de protection du français, par exemple, mais aussi en raison du lien tissé avec l'univers technologique, économique et culturel nordaméricain qui les met en relation avec de multiples réseaux anglophones et augmente sensiblement leur capital social. On ne peut donc pas concevoir le statut de l'anglais au Québec simplement comme celui d'une langue minoritaire. À bien des égards, cette langue hiérarchise ; ceux et celles qui la maîtrisent ont un avantage certain, dans bien des domaines de la vie politique ou des affaires, sur ceux qui ne la parlent pas ${ }^{11}$.

L'analyse de cet aspect de la situation minoritaire du Québec ne se limite pas à la position des communautés anglophones historiques du Québec. Dès lors

10 Terme utilisé par Abdellali Hajjat dans son ouvrage Immigration postcoloniale et mémoire, Paris, L'Harmattan, 2005.

11 II est tout à fait possible pour une personne unilingue anglophone d'accéder aux sphères du pouvoir économique et technologique au Québec, mais pas pour une personne unilingue francophone. 
que la langue et la culture anglaises ouvrent des portes sur l'espace nord-américain on peut penser qu'un calcul rationnel conduise les immigrants - surtout s'ils doivent abandonner leur langue et leur culture d'origine - à choisir de s'intégrer à la culture anglophone plutôt qu'à la culture francophone, tout en vivant au Québec, et à bénéficier d'un potentiel économique supérieur et d'un accès plus facile aux réseaux sociaux des élites.

L'autre clivage est le clivage religieux. Les musulmans du Québec ne sont qu'une petite minorité numérique, traversée par des clivages identitaires (sunnites/chiites, différents groupes ethniques) ainsi que par des courants idéologiques divers. Dans ce cas, deux autres systèmes hiérarchiques qui minorisent la majorité québécoise francophone historique peuvent être interpellés et permettre aux membres de la minorité musulmane de se connecter - symboliquement, politiquement et de façon organisationnelle - à des majorités plus larges que celle du Québec. Le premier système est celui des rapports entre le Québec et le reste du Canada, dans lequel les immigrants peuvent s'insérer en tant qu'immigrants, sans égard à leur ethnicité ou à leur appartenance religieuse, et dont nous avons parlé plus haut. Le deuxième système hiérarchique est celui de la Oumma, à laquelle certains d'entre eux choisissent de s'identifier, et dont l'attraction est fonction de facteurs supranationaux. Dans la minorité musulmane une mouvance s'en réclame, qui rappelle avec fierté qu'elle regroupait près de $23 \%$ des citoyens du monde en 2014 , et que la forte progression du nombre de musulmans est une preuve de la véracité de son message religieux et de sa supériorité morale sur l'Occident. Ce discours, très présent au sein des institutions religieuses se manifeste, dans ce contexte, à la fois sur un plan politique et sur celui de pratiques religieuses conservatrices. On remarquera que ces dernières ne sont apparues dans la culture religieuse de plusieurs pays arabo-musulmans qu'au cours des trente dernières années, sous la poussée du conservatisme wahhabite, parrainé par les monarchies pétrolières, surtout celle de I'Arabie Saoudite.

La connexion symbolique et émotive avec cette entité supranationale peut apporter certains avantages (financement des écoles ou des mosquées, notamment, ou participation à des réseaux transnationaux), mais elle permet surtout de comprendre en partie les processus de différenciation à l'œuvre, qui ne sont pas réductibles à une hiérarchisation locale ${ }^{12}$.

\section{Le différentiel de pouvoir}

Les attitudes de "tolérance " par rapport aux comportements minoritaires sont généralement considérées comme le reflet d'une grande ouverture morale de la part des "tolérants ". Dans un texte intitulé Critique de la tolérance pratique, cependant, Ghassan Hage montre comment les attitudes de tolérance se fondent sur les mêmes clivages hiérarchiques que les attitudes d'intolérance:

"[...] ceux à qui l'on demandait, et à qui l'on demande encore, d'être tolérants, conservent la capacité de se montrer intolérants, ou, pour dire les choses autrement, que les exhortations à la tolérance laissaient intact le pouvoir de ces gens de pratiquer l'intolérance ". (Hage, 2000: 113)

12 Précisons que nos commentaires concernent essentiellement des courants fondamentalistes qui constituent une minorité au sein d'une minorité. 
En d'autres termes, la tolérance elle-même est l'une des facettes des rapports de pouvoir. À cette analyse, à laquelle nous souscrivons, nous souhaitons ajouter une autre dimension, celle du différentiel de pouvoir - implicite, non dit, peut-être même inconscient - entre la majorité et les minorités " tolérées ". Plus une majorité est puissante, plus la capacité de remise en question de son pouvoir politique par des minorités reste lointaine. Elle peut donc se permettre d'être extrêmement tolérante, puisque son pouvoir d'être intolérante demeure. Ainsi, contrairement à une idée dominante dans les publications académiques du Canada anglais, nous estimons que le Québec ne se distingue pas du reste du Canada par une tolérance moins grande envers ses minorités. Il est néanmoins plus conscient de sa vulnérabilité, du fait justement qu'il est lui-même en minorité au sein d'une entité politique qui détient le pouvoir réel, et avec laquelle ses minorités peuvent s'allier politiquement. Les "limites " de la tolérance de la majorité québécoise se manifestent donc plus rapidement que celles de la majorité canadienne, plus confortablement assise sur son pouvoir.

\section{Les critères de définition de la majorité}

S'il est relativement facile de définir une minorité, la majorité est plus difficile à cerner formellement, même si l'intuition nous permet de la saisir à peu près. Voutat et Knuesel parlent du " [...] caractère incertain de ce que recouvre, selon I'expression de Gérard de Rham, le 'noyau dur du bloc au pouvoir' ". Parlant de la Suisse, ils citent François Masnata et Claire Rubattel :

"Par simple négation des critères minoritaires, mais en réunissant cette fois tous les critères sur ce seul groupe [i. e. le "noyau dur»], on peut en effet voir qu'il s'agit des bourgeois des régions développées, masculins, protestants, alémaniques et d'âge mûr.

Il est évident que ce groupe n'est nullement majoritaire d'un point de vue statistique. mais son statut dominant dans la formation sociale lui permet d'être considéré comme la normalité nationale par excellence, la référence obligée de tous les autres groupes [...] "

(Masnata et Rubattel, 1991 : 122).

L'exemple suivant illustre la difficulté de définir la majorité. Quand des femmes appartenant à des courants intégristes revendiquent le droit d'enseigner à des enfants avec le visage caché, comment appréhender la majorité et la minorité qui s'opposent sur cette question ? Faut-il formuler l'interdiction comme étant une interdiction faite " aux femmes musulmanes " par la " majorité " québécoise ? Toutes les femmes musulmanes ne revendiquent pas le voile intégral, une partie des immigrantes ont d'ailleurs quitté leur pays justement pour fuir l'islam radical. Elles ne se considèrent pas lésées par une telle mesure. De plus, beaucoup d'immigrants, musulmans ou pas, catholiques ou pas, seraient d'accord avec l'interdiction du voile intégral. Ne faut-il pas les compter dans la " majorité " qui s'oppose à la " minorité " sur cette question ? II serait erroné, en conséquence, de penser cette question comme une confrontation entre "la minorité musulmane " et la " majorité blanche et catholique ". Le clivage ne se décline pas en termes ethnoculturels, mais bien en termes idéologiques et il traverse de l'intérieur tous les groupes ethnoculturels, y compris la majorité nationale dont une partie a épousé l'idéologie multiculturaliste. Donc la ligne de clivage ne suit pas la démarcation classique entre majorité et minorité. La différentiation n'est pas l'œuvre du groupe majoritaire, mais un choix ancré dans la culture minoritaire en question. II reflète une hiérarchisation morale inversée, dans le sens où les courants qui 
prônent le port du voile intégral affirment aussi qu'ils sont moralement supérieurs à la société occidentale "dépravée ". Le paradigme dominant en sciences sociales, qui analyse cette question en termes d'une majorité créant la différentiation entre elle et la minorité musulmane dans le cadre d'un processus de hiérarchisation ne $s^{\prime}$ applique pas dans le cas décrit, et dans bien d'autres cas de même nature. Or c'est ainsi qu'il est pensé et que les argumentaires pour défendre la " minorité " sont articulés et transformés en mobilisations politiques pour l'égalité.

Pour ceux et celles qui ont adopté cette polarisation comme paradigme, les prises de positions contre l'intégrisme de la part de femmes musulmanes, telle I'ex-députée libérale Fatima Houda-Pépin, ou de l'écrivaine Djemila Benhabib, sont des anomalies. Ces femmes questionnent et perturbent les discours dominants au sein de l'islam, et elles remettent en question les polarisations simples qui départagent les musulmans des non-musulmans. La façon de traiter ces anomalies consiste bien souvent à les qualifier de fausses musulmanes, ou de colonisées, ou d'opportunistes qui veulent monter dans la hiérarchie blanche, ou encore de " poupées Barbie de l'islam light ». Cette dernière remarque, due à Vincent Geisser (2008), a le mérite de mettre en lumière la façon dont l'opposition de ces femmes à l'intégrisme est instrumentalisée dans le discours européen dominant sur l'islam, dans un processus de hiérarchisation sélectionnant les " bons " musulmans (modernistes, laïcs) et les départage des " mauvais " (les conservateurs, les pieux). Et dans certains cas, tel celui de Irshad Manji (2006) la caractérisation proposée par Geisser est sans doute éclairante. Mais, elle ne résout pas deux problèmes de fond. D'abord, celui de la détermination de la ligne de démarcation entre la majorité (qui souhaite des politiques opposées à l'islamisme et confond parfois, dans cette lutte idéologique et politique, islam et islamisme) et la minorité qui souhaite voir une place plus grande accordée à l'expression de la pratique religieuse dans l'espace public, minorité qui ne coïncide pas nécessairement avec le groupe désigné par le terme "les musulmans/musulmanes ". Les prises de position brouillant la représentation de ce clivage sont donc écartées et délégitimées. L'autre problème de fond non résolu par cette approche est celui de la façon de concevoir l'opposition à l'islamisme à partir d'une perspective musulmane. En réalité, la caractérisation de " poupées Barbie " concerne plus l'instrumentalisation de la parole de ces femmes que leur place réelle dans le débat. Elle décrit ce que le discours dominant en a fait, plutôt que ce qu'elles sont, ou que le sens de leur lutte.

La difficulté soulevée dans les paragraphes précédents provient du fait qu'on pense les deux termes de " majorité " et de " minorité " en termes de groupes sociaux délimités par des critères ascriptifs (critères relatifs à l'origine ethnique ou nationale, critères religieux ou culturels) plus ou moins clairs pour une minorité spécifique, plutôt flous pour la " majorité ". Même l'approche intersectionnelle ne serait d'aucun secours pour résoudre cette difficulté, car, centrée sur l'intersection entre " genre/race/classe ", elle ne permet pas de conceptualiser adéquatement les enjeux des clivages idéologiques liés à l'islamisme contemporain. Or la spécificité de cette question se trouve dans l'objectif des courants idéologiques islamistes de devenir hégémoniques dans les communautés musulmanes et de présenter le refus des accommodements comme de l'islamophobie. Le paradigme classique qui démarque les minorités ethno-religieuses des majorités par des critères ascriptifs ne permet pas de déconstruire ce type de discours, il a plutôt tendance à le légitimer voire le renforcer. 
En dépit de la difficulté exprimée dans la citation de Masnata et Rubattel (voir supra), elle propose cependant un critère intéressant pour fonder une définition de la majorité, celui de la perception du " noyau dur " comme représentant une certaine " normalité ". Les réflexions ci-dessous sont une première tentative, de nature exploratoire, allant dans ce sens.

Une définition qui n'enferme pas les groupes dans des critères ascriptifs et qui ouvre des perspectives sur les dimensions institutionnelles et normatives nous semble plus pertinente et plus adaptée au contexte sociopolitique contemporain, surtout dans les sociétés multiculturelles où la diversité est reconnue, célébrée même, et où des brèches dans les " plafonds de verre " se font plus courantes. Elle est fondée non pas sur des marqueurs ethnoculturels ou des phénotypes visibles (religion, langue, couleur de la peau, etc.), mais sur le système institutionnel et normatif sous-jacent auquel un groupe se réfère. Ainsi, même si au Québec ceux qui ont réellement accès au pouvoir politique et économique, le noyau dur du pouvoir, constituaient une minorité au sens de Masnata et Rubattel, il n'en demeure pas moins qu'une majorité de citoyens du Québec s'identifie aux institutions représentatives et aux normes qui fondent ces institutions. La majorité, dans le cadre d'un clivage qui l'oppose à des courants idéologiques minoritaires, peut donc être définie comme étant formée des citoyens qui se reconnaissent dans le système institutionnel et normatif dominant.

Définir la minorité " musulmane " comme une unité de base pour l'analyse de l'exclusion sociale ou de l'assignation identitaire ne permet pas des analyses rigoureuses, car les divers courants idéologiques qui traversent ce groupe amènent les individus s'en réclamant à se positionner très différemment sur des questions clés : la laïcité, les accommodements religieux à accorder ou pas, etc. De plus, la différentiation économique au sein des communautés musulmanes (nous employons le pluriel à dessein) est marquée, particulièrement au Québec et au Canada. Sur les critères de la diplomation, des revenus, ou du prestige social, les communautés musulmanes du Québec sont extrêmement diversifiées. La marginalisation touche certains sous-groupes précis, en fonction de divers processus (dont la discrimination pure et simple, mais pas seulement). En conséquence, la " majorité " devient difficile à déterminer : est-elle constituée par les seuls Québécois historiquement francophones? Et les autres migrants qui s'identifient à la laïcité sans faire partie de la majorité historique, où sont-ils ? Et ceux parmi les immigrants dont le choix s'est porté sur l'intégration en tant que citoyens, reconnus comme citoyens à part entière, sans égard à leurs origines ou à leur apparence ? Et les musulmans anti-fondamentalistes ? Tandis qu'en revenant au système normatif définissant le groupe, on peut identifier clairement la minorité qui réclame que l'espace public accommode les pratiques fondamentalistes, et l'opposer à la majorité (toutes origines ethniques et religieuses confondues) qui se reconnaît dans un système laïc où les principes des droits individuels et collectifs sont reconnus, où le droit de croire tout comme celui de ne pas croire est protégé.

Quand on se situe dans une perspective de lutte pour l'inclusion, la meilleure façon de conceptualiser et de nommer les clivages et les groupes marginalisés n'est pas évidente. Faire des mobilisations en fonction de l'idée de " race ", par exemple, peut avoir comme dommage collatéral de reproduire, dans la culture dominante, les clivages selon lesquels la marginalisation opère. Le débat lancé 
par Anthony Appiah et Amy Gutmann en 1998 sur la pertinence politique du recours explicite à la notion de race dans l'analyse de l'exclusion et dans les luttes pour l'inclusion reste d'actualité, surtout quand on le transpose aux marqueurs ethnoculturels.

Les remarques précédentes n'excluent pas toutefois qu'un idéal normatif comme celui de la laïcité, notamment, soit instrumentalisé et qu'il devienne effectivement un cheval de bataille pour l'islamophobie. Sur ce point, il faut faire une distinction nette entre des concepts analytiques convoqués dans la théorisation d'un phénomène, et les instrumentalisations de ce concept dans I'usage courant, par divers groupes d'intérêts, dans le cadre de luttes politiques. Le concept de laïcité n'est pas le seul à être instrumentalisé : les concepts de " démocratie " et de "droits humains " le sont tout autant, ce qui ne veut pas dire qu'il faille les délégitimer comme objectifs à atteindre. L'instrumentalisation d'une norme par des acteurs politiques ne signifie pas qu'elle soit automatiquement à rejeter.

\section{Le rôle du groupe jugé minoritaire dans l'édification des clivages}

Quand nous disons que la différentiation est liée à un processus de hiérarchisation, il ne faut pas en conclure automatiquement que c'est la " majorité " qui entreprend d'ériger des critères de différenciation marginalisant les minorités. Le groupe dominant n'a pas, seul, le pouvoir de déterminer quels marqueurs vont entrer en jeu, et s'ils ont des connotations positives ou négatives. Car, au-delà de leur capacité de "renverser le stigmate " et de transformer le sens d'un marqueur, les groupes dits minoritaires peuvent choisir de mettre en avant des marqueurs qui les distinguent et qui se sont développés ailleurs, dans d'autres logiques que celle de leur situation de minoritaire.

Revenons au cas de l'islam et des musulmans. On ne peut pas dire, comme le veut la théorie constructiviste du fait minoritaire, que le clivage est constitué dans le rapport lui-même, car c'est dans d'autres contextes sociaux, que les marqueurs du clivage (ainsi le hidjab) ont été construits. Si la plupart des musulmans du Québec se considèrent comme membres à part entière de la société québécoise et considèrent que leur appartenance à l'islam est personnelle et non pas politique ${ }^{13}$, il reste qu'une minorité parmi les musulmans valorise ce clivage et vise à le renforcer et à le promouvoir. II faut analyser l'érection de ces clivages comme une lutte pour l'hégémonie à l'intérieur des communautés musulmanes. Les marqueurs sont alors vus comme une façon de préserver la communauté des influences néfastes de la société d'accueil ${ }^{14}$, perçue comme étant moralement dépravée. On pourrait voir dans le discours de certaines forces politiques à l'échelle des sociétés musulmanes de telles intentions qui se matérialisent par la création d'écoles islamiques et de mosquées. L'établissement de lieux de culte est un droit fondamental à préserver. Du point de vue de l'analyse, cependant, peut-on ignorer la façon dont ils sont instrumentalisés dans les luttes idéologiques qui se développent dans les sociétés

13 Cette majorité de musulmans peut très bien combiner appartenance à la société d'accueil avec une attitude fortement ou faiblement pratiquante, ou non pratiquante, ou même non religieuse.

14 Cet aspect a été bien documenté dans la recherche-terrain de Ali Daher (1988). 
musulmanes, et la façon dont elles se sont traduites en conflits politiques violents ? Or ceci pose des problèmes éthiques et méthodologiques difficiles, sinon impossibles à résoudre. Aucune étude anthropologique ne pourrait produire, par exemple, la riche analyse qu'a faite le journaliste lan Johnson dans Une mosquée à Munich (Johnson, 2011) ) $^{15}$. Ni les paradigmes théoriques ni les méthodes classiques de recherche en sciences sociales $n^{\prime}$ auraient pu orienter le regard vers ce que Johnson a identifié dans le rôle social et politique de ce lieu de culte en situation de migration car sa recherche s'apparente plus à une enquête policière historique qu'à une recherche scientifique. Cependant, ce que met en évidence cet auteur contribue à la compréhension des processus de construction des rapports entre les communautés musulmanes européennes et les sociétés d'accueil.

Une dynamique résulte de l'intersection de clivages religieux et idéologiques : des revendications non raisonnables et quelquefois irritantes ${ }^{16}$ sont mises en avant par une petite minorité militante. À cause d'une médiatisation sensationnaliste ${ }^{17}$, non exempte de stéréotypes essentialistes et de calculs politiques (Antonius, 2013), ces revendications provoquent des réactions dans le groupe majoritaire qui peuvent relever en partie de l'islamophobie. II s'ensuit un mouvement de solidarité et d'identification parmi les musulmans, - et ce même quand ils n'approuvent pas nécessairement ces revendications -, qui estiment qu'il faut les accommoder, car le refus de le faire serait considéré comme islamophobe.

La demande de musulmans d'interrompre le travail, pour faire les prières à des heures bien déterminées, donne une autre illustration du rôle du groupe minoritaire dans l'édification des clivages. Nous ne rentrerons pas dans le débat théologique concernant les obligations, nous constatons seulement que jusqu'à tout récemment, dans la plupart des pays musulmans, ni les institutions publiques ni les institutions privées $n^{\prime}$ interrompaient le travail. Or cette exigence est présentée par ceux qui la portent comme une injonction religieuse non sujette à discussion. D'un point de vue multiculturaliste, les objections à son acceptation seraient considérées comme un signe d'intolérance ou de discrimination et seraient très malvenues. Si de telles pratiques devaient être adoptées par une proportion importante de musulmans en situation de migration, ceci entraînerait des modifications importantes dans le fonctionnement des organisations, des écoles et des universités, et elles contribueraient à instaurer un clivage de plus entre les musulmans et la " majorité ", lequel ne relève pas d'une hiérarchisation construite par la " majorité ". L'effort de propagande systématique et massive de la part des monarchies pétrolières durant les quarante dernières années a

15 Ce livre est le résultat d'une enquête historique sur des opérations clandestines, d'envergure internationale, s'étendant sur soixante-dix ans, autour du contrôle politique du conseil d'administration de la mosquée et de ses rapports avec les autorités allemandes.

16 Nous parlons de revendications promues par les courants wahhabites qui étaient, jusqu'à tout récemment, largement considérées comme inacceptables dans la plupart des sociétés musulmanes urbanisées: le voile intégral, la ségrégation sexuelle dans les amphithéâtres à I'université, I'interdiction de la musique à la maternelle, etc.

17 On pourrait citer la question du voile intégral. Le résultat est que le clivage entre la majorité et les musulmans s'est trouvé renforcé autant à cause de l'action du groupe majoritaire que celle de courants politiques au sein du groupe minoritaire. 
favorisé les interprétations extrêmement rigides des obligations religieuses ${ }^{18}$, et les courants extrémistes ont graduellement occupé une position importante dans les sociétés musulmanes, favorisant l'émergence de courants salafistes et djihadistes ${ }^{19}$. On peut dès lors interpréter l'action de ceux qui militent pour la généralisation de pratiques rigides au sein des communautés musulmanes comme une tentative d'instaurer une hégémonie sur ces communautés. Là encore, la différentiation qui s'instaure entre la société majoritaire et cette minorité, trouve son origine au sein du groupe minoritaire.

\section{Logiques majoritaires/positions minoritaires}

Le paradigme constructiviste centré sur la hiérarchisation ne permet pas de saisir l'existence, au sein de groupes minoritaires, de discours dont la logique appartient aux majoritaires dans d'autres contextes que celui de l'immigration, et qui ont été transposés et articulés à une position de minorité démographique.

Cet aspect est particulièrement visible dans le cas des discours fondamentalistes islamistes. Une de leurs particularités est qu'ils sont devenus dominants dans les sociétés arabo-musulmanes, et qu'ils se manifestent par des pratiques religieuses, marginales il y a quelques décennies, courantes et même banales aujourd'hui. En d'autres termes, ces courants idéologiques ont réussi à transformer des comportements liés à une lecture littérale du dogme en symboles identitaires, repris et revendiqués par de nombreux musulmans, qui ne partagent pas nécessairement les justifications et l'idéologie sur lesquels ils sont fondés. Le hidjab en est un exemple. Nous interprétons les processus qui ont produit cette évolution comme des logiques majoritaires, c'est-à-dire des logiques d'action visant à différencier les musulmans, là où ils sont majoritaires, des non-musulmans par des marqueurs omniprésents, qui de surcroît opèrent une hiérarchisation des premiers sur les derniers. Le paradigme dominant du rapport majorité/minorité et les processus de différenciation/hiérarchisation jouent un rôle majeur dans les sociétés de référence des migrants. La construction du clivage par des marqueurs visibles est ainsi transformée en obligation religieuse. Transposée en situation de migration et portée par une culture devenue minoritaire, les traces de sa logique majoritaire d'origine ne sont plus visibles. Le paradigme reste donc valable, mais les processus de différenciation/ hiérarchisation qu'il modélise opèrent au sein du groupe minoritaire, lequel se définit en fonction d'une majorité transnationale ${ }^{20}$.

La volonté hégémonique dont nous avons parlé plus haut prend parfois des tournures étonnantes. Au début de septembre 2014, de jeunes gens musulmans de tendance salafiste se sont mis à patrouiller dans les rues de la petite ville de Wuppertal en Allemagne, portant des vestes de couleur orange sur lesquelles

18 Nombre d'historiens spécialistes des sociétés arabes et musulmanes savent que la pratique des rituels religieux est relativement souple et que les règles sont accommodantes.

19 Les dérives de l'islam politique dans les sociétés arabes " post-révoltes " ont permis à certaines voix critiques d'être entendues. On peut signaler la dénonciation de I'influence wahhabite dans les programmes scolaires. Voir à ce sujet Khaled (2014).

20 En amont de ces phénomènes, les rapports coloniaux ont joué un rôle déterminant, mais la forme spécifiquement religieuse qu'a prise la résistance au colonialisme a des conséquences en aval, dans les sociétés où cette résistance est née. 
il était écrit en grands caractères "Shariah Police ", et interpellant les passants aux comportements ne cadrant pas avec les codes rigides de la Charia, surtout aux abords des discothèques. Évidemment, cette initiative a été condamnée par l'ensemble de la classe politique. Comment interpréter ce phénomène, à la lumière des considérations développées ci-haut?

II est possible de rejeter d'un revers de main l'importance du phénomène, en disant qu'après tout il ne s'agit que d'un petit groupe de zélés conservateurs, qu'on retrouve dans toutes les religions. Une tendance théorique multiculturaliste pourrait même admirer leur capacité à défier les normes d'un État qui se

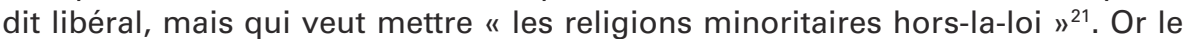
fait que ces individus se sentent l'autorité morale de se positionner en gardiens de l'ordre public, d'un ordre qui est le leur et non pas celui de la majorité, est significatif. C'est un comportement de majoritaires ne tolérant pas d'écart à la norme, qu'ils veulent faire imposer par l'État. Porté par des groupes minoritaires en situation de migration, ce comportement n'est cependant pas perçu comme résultant d'une logique majoritaire.

Dans toutes les grandes villes européennes comme Londres ou Bruxelles où des communautés musulmanes sont concentrées dans certains quartiers, on a vu des groupes plus ou moins organisés tenter d'interdire dans l'espace public la mixité des sexes par exemple. Dénoncée d'abord par des partis de droite, on a observé qu'il était parfois difficile de blâmer ce type de pratique sans être associé à ces groupes politiques ${ }^{22}$.

Cet incident illustre un aspect typique de la situation actuelle : des courants idéologiques eux-mêmes minoritaires au sein de groupes minoritaires ont la capacité de faire changer les règles régissant l'usage de l'espace public. L'incident aurait des conséquences plutôt symboliques s'il s'agissait de minorités isolées, dont la culture authentique est menacée de disparition. Or c'est loin d'être le cas, puisque des demandes similaires sont exprimées dans diverses capitales européennes, et que des réseaux transnationaux permettent d'organiser et de coordonner ce type de demandes. Une partie de cette organisation se faisant de façon clandestine, elle échappe aux recherches académiques. Aucune recherche de type anthropologique ou interculturelle sur la problématique de radicalisation n'aurait pu détecter (et encore moins prédire) le départ possible de 900 à 1000 djihadistes français pour aller combattre au sein des groupes islamistes en Irak et en Syrie en 2013-2014.

Le rapprochement entre la radicalisation violente et la radicalisation sociale " soft " ne nous semble pas farfelu, puisque les mêmes bases dogmatiques sont au cœur de ces deux types de radicalisation, et que l'une est une condition nécessaire - heureusement pas suffisante - de l'autre. Les projets de résurrec-

\footnotetext{
21 Colloque tenu à I'université de Sherbrooke en octobre 2014, avec pour titre "La religion hors-la-loi : I'État libéral à l'épreuve des religions minoritaires ".

22 Un cas de réaction aberrante à cette situation doit cependant être rappelé : I'association des universités de Grande-Bretagne, Universities UK, a produit un guide à l'intention des universités membres, les encourageants, si besoin, à pratiquer la ségrégation dans leurs amphithéâtres lors d'événements publics, "afin de ne pas exclure " les femmes musulmanes (Universities UK, 2013). Devant le tollé suscité par cet avis, I'association I'a toutefois suspendu temporairement.
} 
tion de la Oumma ou du Califat ne sont pas des demandes minoritaires. Elles procèdent d'une volonté de domination d'un groupe majoritaire, même si elles sont portées par des tendances elles-mêmes minoritaires au sein des groupes minoritaires en situation de migration.

\section{Conclusion : les musulmans et I'islam comme phénomène minoritaire}

L'intégration des communautés et des individus issus de l'immigration en provenance de pays à majorité musulmane soulève des enjeux analytiques, éthiques et politiques, difficiles à cerner simultanément, car ils renvoient à des niveaux d'analyse différents qu'il n'est pas aisé de combiner. Chaque paradigme théorique adopté pour l'analyse de cette question permet d'identifier certains des enjeux, en laissant d'autres dans l'ombre, et entraînant des positions politiques qui ne font pas l'unanimité, même au sein de groupes qui partagent des orientations communes. Que l'on pense, par exemple, aux clivages qui ont divisé le mouvement féministe sur la question du hidjab.

Sur cette question, nous avons tenté d'examiner les difficultés du recours à un paradigme dominant en sciences sociales, celui qui place les rapports de pouvoir entre majorité et minorités au cœur de l'analyse des rapports ethniques, et qui les fonde sur un double processus de différenciation/hiérarchisation visant à affirmer un rapport de domination de la majorité sur la minorité. Deux conclusions générales se dégagent de l'analyse.

La première est que le rapport majorité/minorité se complexifie lorsqu'on considère qu'une majorité à une échelle donnée peut être une minorité à une autre échelle, ce qui est le cas du Québec. Cette conclusion n'a rien de nouveau, mais nous en avons tiré une autre, moins évidente : on peut être une " minorité " dans l'espace québécois, mais bénéficier d'une position de majoritaire si on se situe dans un autre espace, I'espace canadien, et que l'on s'identifie à la majorité canadienne. C'est-à-dire que la " majorité " québécoise est mise dans une situation de minorisation simultanément par le haut (le niveau fédéral et ses institutions) et par le bas, par des "minorités " qui s'insèrent dans les rapports de pouvoir du côté de la majorité au niveau canadien, bénéficiant du coup d'avantages dont jouissent les groupes majoritaires.

Nous avons aussi examiné plus spécifiquement le cas des minorités musulmanes au Québec. Celles-ci ne constituent pas une communauté, mais un ensemble de communautés, ainsi qu'un ensemble d'individus qui pourraient choisir de s'insérer dans l'espace québécois comme citoyens avant tout et non pas comme membres d'une communauté musulmane. Ces groupes sont traversés par des courants idéologiques minoritaires, mais qui, du fait de leurs liens transnationaux, se connectent à des groupes plus larges et reproduisent des processus de différenciation/hiérarchisation dont la logique est avant tout une logique de majoritaires, rendue possible par les particularités de la montée de I'islam politique - et de ses dérives - à l'échelle mondiale.

C'est la combinaison de ces deux facteurs qui nécessite que le paradigme dominant de la sociologie des minorités, qui met les processus de différenciation/hiérarchisation au cœur du rapport de domination, soit ajusté en fonction 
des contextes précis où il est interpellé. Car il faut tenir compte de multiples niveaux nationaux et transnationaux qui s'imbriquent les uns dans les autres, qui renversent les rapports de domination à l'occasion, et qui nous obligent à intégrer, dans l'analyse du fait minoritaire en situation de migration, l'évolution des courants idéologiques dans les pays d'origine des migrants, et leurs conceptions de l'altérité et de la hiérarchisation.

\section{Références bibliographiques}

Antonius Rachad (2012) La polarisation du discours sur l'islam en Occident : quelques réflexions, in M. Labelle, J. Couture et F. Rémiggi Éds., La Communauté politique en question : Regards croisés sur l'immigration, la citoyenneté, la diversité et le pouvoir, Québec, Presses de l'université du Québec, pp. 145-169.

Antonius Rachad (2013) A Mediated Relationship: Media Representations of Muslims and Arabs as a Political Process, in Jenna Hennebry and Bessma Momani Éds., TargetedTransnationals: The State, the Media, and Arab Canadians, Vancouver, University of British Columbia Press, pp. 110-128.

Appiah K. Anthony and Gutmann Amy (1998) Color Conscious: The Political Morality of Race, Princeton, Princeton University Press, 200 p.

Assemblée nationale du Québec (2013) Charte affirmant les valeurs de laïcité et de neutralité religieuse de l'État ainsi que d'égalité entre les femmes et les hommes et encadrant les demandes d'accommodement, Projet de loi 60, 1 re session de la 40 e législature.

Boucher Jacques L. et Thériault Joseph-Yvon (2003) Petites sociétés et minorités nationales : enjeux politiques et perspectives comparées, Québec, Presses de I'université du Québec, 420 p.

Chevrier Marc (2012) La république québécoise. Hommages à une idée suspecte, Montréal, Éditions du Boréal, 416 p.

Daher Ali (1998) La construction de l'islamité et l'intégration des musulmans au Québec dans le discours de leurs leaders, Thèse de doctorat, université du Québec à Montréal.

Geisser Vincent (2008) Les poupées Barbie de l'islam light : exhibitionnisme et érotisme victimaires, [en ligne] consulté le 12 septembre 2014. URL : http:// oumma.com/Les-poupees-Barbie-de-I-Islam

Gouvernement du Canada (2006) Les Québécois, Débats de la Chambre des communes, 141 (086), 1re session, 39e législature, Compte rendu officiel (Hansard), le vendredi 24 novembre.

Guillaumin Colette (1972) L'idéologie raciste, genèse et langage actuel, Paris/La Haye, Mouton, 378 p.

Guillaumin Colette (1992) Sexe, Race et Pratique du pouvoir. L'idée de Nature, Paris, Côté-femmes, 239 p.

Hage Ghassan (2000) Critique de la tolérance, pratique, in Caroline Mackenzie Éd., Australie, Autoportraits, La Tour d'Aigues, Éditions de I'aube, pp.105-126.

Hajjat Abdellali (2005) Immigration postcoloniale et mémoire, Paris, L'Harmattan, $150 \mathrm{p}$. 
Institut Simone de Beauvoir (2010) Déclaration de l'institut en réponse au projet de loi 94, [en ligne] consulté le 12/09/2014. URL : https://www.concordia.ca/ content/dam/artsci/sdbi/docs/positions/SdBI2010Bill94-bilingualresponse.pdf

Johnson lan (2011) Une Mosquée à Munich. Les nazis, la CIA et la montée des Frères musulmans en Occident, Paris, J.C. Lattès, 380 p.

Khaled Ahmed (2014) Hakatha zouri'a al fikr al takfiri fi tounes, (C'est ainsi que la pensée takfiriste a été implantée en Tunisie),Tunis, Dar Zakharef.

Korteweg Anna C. (2006) The Sharia Debate in Ontario, ISIM Reviews, [en ligne] consulté le 12/09/2014. URL : https://openaccess.leidenuniv.nl/handle/1887/17094

Labelle Micheline (2003) À propos de la reconnaissance de la diversité dans I'espace national québécois. Exclusion ou incorporation segmentée ?, in L. Boucher et J.-Y.Thériault, Petites sociétés et minorités nationales : enjeux politiques et perspectives comparées, Québec, Presses de l'université du Québec, pp.27-47.

Labelle Micheline (2010) Racisme et Antiracisme au Québec. Discours et déclinaisons, Québec, Presses de l'université du Québec à Montréal, 212 p.

Manji Irshad (2006) Musulmane mais libre, Paris, Le Livre de Poche, 279 p.

Masnata François et Rubattel Claire (1991) Le pouvoir suisse, Lausanne, L'Aire, 319 p.

Razack Shereen (1998) Looking White People in the Eye: Gender, Race, and Culture in Courtrooms and Classrooms, Toronto, University of Toronto Press, $272 \mathrm{p}$.

Razack Sherene H. (2011) La chasse aux Musulmans. Évincer les Musulmans de I'espace politique, Montréal, Lux Editeur, 352 p.

Simon Pierre-Jean (2006) Pour une sociologie des relations interethniques et des minorités, Rennes, Presses universitaires de Rennes, 347 p.

Universities UK (2013) External Speakers in Higher Education Institutions [en ligne] consulté le 5/09/2012, URL : http://www.universitiesuk.ac.uk/highereducation/Pages/Externalspeakersinhighereducationinstitutions.aspx\#.VBXfOee9nd5

Voutat Bernard et Knuesel René (1997) La question des minorités. Une perspective de sociologie politique, Politix, 10 (38), pp. 136-149.

Wirth Louis (1945) The Problem of Minority Groups, in Ralph Linton Ed., The Science of Man in the World Crisis, New York, Columbia University Press, pp. 347-372 


\section{Rachad Antonius}

\section{"..? Repenser les catégories de " majorité " et de " minorité " : I'islamisme comme phénomène minoritaire dans les sociétés occidentales}

Les politiques d'intégration sont généralement pensées, justifiées et analysées en faisant référence, explicitement ou implicitement, aux catégories de minorité et de majorité. Le rapport entre les deux catégories est considéré comme fondateur du cadre régissant la gestion de la diversité. Or, bien que fondamental, ce rapport est à questionner d'un point de vue théorique et empirique. De plus, les flux de personnes et d'informations font que des minorités locales entretiennent des liens avec des majorités à des niveaux nationaux et transnationaux, et que des majorités locales peuvent se trouver en situation de minorité. Ceci est particulièrement vrai dans le cas des "petites nations " comme le Québec. L'article a pour objectif de problématiser le rapport majorité/minorité, les processus sociaux de différenciation et de hiérarchisation et les liens transnationaux qui mettent en relation des minorités locales avec des discours majoritaires. Le cas des musulmans au Québec servira de référence à cette réflexion.

\section{... Rethinking the categories of 'majority' and 'minority': Islamism as a minority phenomenon in Western societies}

Integration policies are usually conceived, justified and analyzed with explicit or implicit reference to notions of majority and minorities. The relationship between the two categories is considered to be the foundation of diversity management. However, while fundamental, this relationship is problematic in several respects, because various factors contribute to unsettling it. Moreover, transnational flows of people and information link local minorities with majorities elsewhere, and local majorities may simultaneously be minorities at another level. This is particularly true in the case of "small nations" such as Quebec. The article aims at problematizing this majority/minority relationship, the social processes of differentiation and hierarchisation that comes with it, and the transnational ties that connect local minorities with majority discourses elsewhere. This reflection will be grounded on the case of Muslims in Quebec.

\section{Migraciones temporales del trabajo y relaciones mayoría/ minorías en Canadá}

A partir de un enfoque crítico de las políticas públicas canadienses de inmigración y de empleo, este artículo presenta un estudio comparativo internacional sobre las consecuencias socioeconómicas locales de los flujos de trabajo temporal migrante en materia de empleo y de acceso a los derechos sociales laborales. El estudio examina las incidencias locales respecto de la relación mayoría/minorías de los principales programas canadienses de "trabajadores extranjeros temporales». Desde la perspectiva de la división internacional del trabajo, el texto analiza cómo estos flujos se imponen como figura central y pone de relieve sus consecuencias, especialmente en lo que se refiere a la desintegración de la relación salarial y a las restricciones de acceso a los derechos sociales. La conclusión se centra en el papel ambivalente del Estado en la gestión de estos flujos, así como en las incidencias sobre la relación mayoría/minorías de este fenómeno, cuya envergadura internacional no puede ocultar las pesadas repercusiones locales. 